\title{
Spinal versus general anaesthesia in percutaneous nephrolithotomy
}

\author{
Wani $\mathrm{Z}^{1}$, Harkawat D.K. ${ }^{2}$, Sharma $\mathrm{M}^{3}$ \\ ${ }^{1}$ Dr. Zara Wani, Post Graduate, ${ }^{2}$ Dr. Dev Kumar Harkawat, Assistant Professor, ${ }^{3}$ Dr Meenaxi Sharma, Professor and \\ Head, all authors are affiliated with Department of Anaesthesiology \& Critical Care, NIMS Medical College \& Hospital, \\ Jaipur, India
}

Address for correspondence: Dr. Zara Wani, Email: zarawani14@yahoo.com

\begin{abstract}
Background: Hemodynamic stability and blood loss reduction are subjects to further consideration in patients undergoing percutaneous nephrolithotomy (PNCL). Objectives: This study compared the preference of spinal anaesthesia (SA) or general anaesthesia (GA) in respect to mentioned concerns. Methods: In this randomized clinical trial, 59 patients who underwent PCNL divided into SA and GA groups. 15-20 mg from intra-thecal bupivacaine $0.5 \%$, and premedication of 0.01-0.02 mg from midazolam, were given to patients in SA group $(\mathrm{n}=29)$. Patients in GA group $(\mathrm{n}=30)$ received premedication of $1-2 \mu \mathrm{g} / \mathrm{kg}$ from fentanyl and $0.01-0.02 \mathrm{mg} / \mathrm{kg}$ from midazolam, and intravenously anaesthetized with $100 \mu \mathrm{g} / \mathrm{kg} / \mathrm{min}$ of propofol and $0.5 \mathrm{mg} / \mathrm{kg}$ of atracurium, given by continuous infusion and N2O/O2 $50 \%$. Mean arterial pressure (MAP) and heart rate were recorded intra-operatively and during recovery. Results: MAP and heart rate show no significant differences at designated time points between two groups $(P>0.05)$. Surgery time, anesthesia time, bleeding volume, and analgesic intake were significantly reduced in SA group $(P<0.05)$. Conclusions: Regional epidural anaesthesia is an alternative technique for PCNL which achieves more patient satisfaction, less early postoperative pain and less adverse effects from medication with the same efficacy and safety compared to general anaesthesia. It seems that, in patients undergoing PNCL, SA is as effective and safe as GA and with many advantages over it.
\end{abstract}

Keywords: Percutaneous nephrolithotomy, Anesthesia, Complications

\section{Introduction}

Urinary tract stone disease is a major health-care problem, and after urinary tract infections and prostate pathology, is the third in rank among the diseases of the urinary system. Percutaneous nephrolithotomy (PCNL) is the treatment of choice for large renal calculi, staghorn calculi and calculi which fail treatment with extracorporeal shockwave lithotripsy and ureteral endoscopy [1-3].

PCNL can be performed under general anaesthesia, regional anaesthesia or local anaesthesia. Nowadays, PCNL is usually performed under general anaesthesia due to better control of breathing and more comfort for the patients. However, there are some occasionally side effects from general anaesthesia such as lung atelectasia, drug allergy and postoperative nausea and

Manuscript received: $04^{\text {th }}$ January 2017

Reviewed: $10^{\text {th }}$ January 2017

Author Corrected: $17^{\text {th }}$ January 2017

Accepted for Publication: $28^{\text {th }}$ January 2017 vomiting [4,5]. Recently, PCNL under spinal anaesthesia was reported as having some advantage over general anaesthesia, such as lower post operative pain, lower dose requirement for analgesic drugs, and avoidance of the side effects from multiple medication during general anaesthesia [4-6].

The aim of this study was to compare the efficacy and safety of regional epidural anaesthesia and general anaesthesia in patients who underwent PCNL.

There are controversies among researchers regarding the use of SA in PNCL due to the most important issue which is acute hypotension, resulting from sympathetic block [7-10].

Therefore, BP and pulse rate (PR) can be helpful to monitor sympathetic drive in these patients. There are many studies comparing GA and SA in several surger- 
ies however, there is no definite comparison made by $\mathrm{BP}$ and PR in PNCL during surgery and in recovery room.

\section{Objectives}

Considering the type of anaesthesia as well as patients' hemodynamics that can influence on surgery outcomes and relevant morbidity and mortality of the intervention, and that these factors directly reflect on regional health-care, we aimed this study to compare mean BP and PR among PNCL patients underwent GA and SA.

\section{Patients and Methods}

Subjects-In this randomized clinical trial, all patients coming to NIMS medical college \& hospital as PNCL candidates were included sequentially if they met these inclusion criteria: age between 18-65 years with physical status I or II of American Society of Anaesthesiologists (ASA). All patients with spinal deformity, local infection at injection site, pregnancy, history of any neuromuscular or psychiatric disorder or chronic pain, who were suffering from hypertension, diabetes and coagulation disorders, patients with hypersensitivity to any anaesthesia drugs, substance abusers, and patients who needed anaesthesia higher than T4 and lower than T10 levels were excluded. The included patients were divided into SA and GA groups using randomized number table. Standard monitoring included continuous electrocardiogram, pulse oximetry, and end-tidal carbon dioxide. Non-invasive BP measurements were performed at 5-min intervals. All patients were routed with a green (18-gauge) catheter and infused with 3-4 cc/kg isotonic crystalloids. Maintenance venous liquid during surgery was based on 4/2/1 rule. For blood loss limited to "maximum allowable blood loss", $3 \mathrm{~mL}$ of Ringer solution was injected for every $1 \mathrm{ml}$ of blood loss, and equal volume of matched iso-group packed cell for more blood losses. Both types of anaesthesia were performed by a 4th year resident of anaesthesiology.

GA Group-Premedication of $1-2 \mu \mathrm{g} / \mathrm{kg}$ from Inj Fentanyl and $0.01-0.02 \mathrm{mg} / \mathrm{kg}$ from Midazolam was administered. Oxygen with an inspired fraction of 1.0 was administered for $3 \mathrm{~min}$ before intubation. Then, GA was induced by $2 \mathrm{mg} / \mathrm{kg}$ Inj Propofol, and to obtain desired anaesthesia, $0.5 \mathrm{mg} / \mathrm{kg}$ of Inj Atracurium was injected intravenously for easier intubation; then, all patients were intubated by a suitable endotracheal tube. For maintaining GA, an intravenous $100 \mu \mathrm{g} / \mathrm{kg} / \mathrm{min}$ of
Inj Propofol with 50\% O2 and 50\% N2O were induced. The ventilation protocol consisted of an inspired oxygen fraction of 1.0, Inspiratory to expiratory ratio of $1: 2$, and a respiratory rate adjusted to normocapnia (endtidal carbon dioxide partial pressure between 30 and 40 $\mathrm{mmHg}$ ). Mechanical ventilation has been set with a tidal volume of $10 \mathrm{ml} / \mathrm{kg}$ ideal body weight (IBW) and ZEEP (zero-positive end expiratory pressure). Inj Atracurium and Inj Fentanyl re-administration was based on train-of-four (TOF) and every 45 minutes, respectively.

SA Group- Premedication of 0.01-0.02 mg/kg from Inj Midazolam was administered. The patients were placed in a sitting position. The drug was administered by a $25-$ gauge Quincke needle in midline of L3-L4 or L4-L5 level by a physician. For inducing SA, isobar intrathecal 15-20 mg of Inj Bupivacaine 0.5\% without any additives was administered. Then, the patients' positions were changed to prone and intranasal $100 \%$ oxygen was administered. Sensory blockade was evaluated by a cotton peak (for heat perception) or a needle (for touching sense) every 15-20 seconds; then, motor blockade was tested by Bromage scale with following score: $0=$ no paralysis; $1=$ inability to raise extended leg; 2 = inability to flex knee; 3 = inability to move leg joints. Blood pressure below $100 \mathrm{mmHg}$ of $30 \%$ from the baseline was corrected by $6 \mathrm{mg}$ ephedrine and crystalloids, and all PR descents (less than 60/min) were treated by intravenous Atropine.

Anaesthesia Assessment- Systolic Blood Pressure (SBP), Diastolic Blood Pressure (DBP), Mean Arterial Pressure (MAP), and PR were recorded every 20 minutes during surgery from the beginning of anaesthesia. Intra-operative blood loss was calculated by blood volume of suction devices, and estimated volume of blood in sponges and drapes already were weighted before operation.

SBP, DBP, MAP, and PR were recorded in the PACU, every $10 \mathrm{~min}$ from entering PACU. Fifty $\mathrm{mg}$ from Inj Meperidine was administered in patients suffered from additional pain. All patients were positioned in supine. MAP and PR were evaluated every 10 minutes for 1 hour. Other information were extracted from medical files and inserted into a pre-prepared checklist.

Statistical Analysis- Based on a pilot study in 12 patients (six from each group), we determined that a sample size of 26 in each group would be sufficient to 
detect the differences between mean of blood loss and analgesic demand, estimate a standard deviation of 10 , a power of $95 \%$, and a significance level of $5 \%$; this number was increased to 30 per group, to allow a predicted drop-out of around 10\% from the study.

The data were evaluated and analyzed by SPSS version 19 (SPSS Inc., Illinois, USA). All quantitative data were expressed as mean $\pm \mathrm{SD}$, and qualitative data as No. (\%). For comparing the groups, t-test and MannWhitney-U test were used for parametric and nonparametric data, evaluated by Kolmogorov-Smirnov test, respectively. $\mathrm{P}$ less than 0.05 were considered as significant.

\section{Results}

Demographic Data- Fifty nine patients were enrolled in the study consisting of 38 males and 21 females. The patients were randomly divided into SA $(n=29)$ and GA $(n=30)$ groups. Table 1 demonstrates all demographic data. Surgery duration $(\mathrm{P}=0.016)$ and anaesthesia duration $(\mathrm{P}=0.044)$ were significantly lower in SA (Table 2). According to Bromage scale, motor block level was zero in all patients in SA group.

Table-1: Comparison of Demographics between Two Groups

\begin{tabular}{|c|c|c|c|}
\hline Variable & General Anesthesia & Spinal Anesthesia & P value \\
\hline Gender & & & 0.86 \\
\hline Male, No. (\%) & $19(63.3)$ & $19(65.5)$ & $10(34.5)$ \\
\hline Female, No. (\%) & $11(36.7)$ & $23(79.3)$ & 0.590 \\
\hline ASAaClass & & $6(20.7)$ & 0.022 \\
\hline I & $22(72.3)$ & $39.6 \pm 9.7$ & 0.129 \\
\hline II & $8(26.7)$ & $26.4 \pm 3.8$ & \\
\hline BMIa, Mean \pm SD, $\mathrm{kg} / \mathrm{m} 2$ & $46.9 \pm 13.6$ & & \\
\hline
\end{tabular}

Table-2: Duration of Surgery, Anesthesia, Recovery time, Blood Loss, Analgesic Demand, and Blood Transfusion Amount in Both Groups

\begin{tabular}{|c|c|c|c|}
\hline Variable & General Anesthesia & Spinal Anesthesia & P value \\
\hline Surgery Duration, Mean \pm SD, min & $112.2 \pm 18.3$ & $99.3 \pm 21.1$ & 0.016 \\
\hline Anesthesia Duration, Mean \pm SD, min & $112.2 \pm 18.3$ & $101.3 \pm 22.03$ & 0.044 \\
\hline Recovery Duration, Mean \pm SD, min & $42.2 \pm 12.8$ & $41.5 \pm 19.1$ & 0.878 \\
\hline Blood Loss, Mean \pm SD, ml & $331.7 \pm 151.1$ & $211.03 \pm 89.6$ & 0.001 \\
\hline Analgesicdemand, Mean \pm SD & $6.3 \pm 8.9$ & $2.03 \pm 6.3$ & 0.038 \\
\hline Blood Transfusion, No. (\%) & & & 0.321 \\
\hline Positive & $1(3.3)$ & $0(0)$ & \\
\hline Negative & $29(96.7)$ & $29(100)$ & \\
\hline
\end{tabular}

Endpoint Results- Table 2 demonstrates blood loss, analgesic demand, and blood transfusion amount in both groups. As seen, blood loss $(\mathrm{P}=0.001)$ and analgesic demand $(\mathrm{P}=0.038)$ were significantly higher in GA group.

\section{Discussion}

Percutaneous nephrolithotomy is a minimally invasive surgery which is accepted for treating large renal and upper ureteric calculi [1-3]. Several new techniques of PCNL such as mini-PCNL and tubeless PCNL were reported to decrease morbidity, analgesic requirement and duration of hospitalization [11]. The method of anaesthesia was reported to minimize morbidity following PCNL. The disadvantages of general anaesthesia compared to regional spinal anaesthesia are increased incidence of anaphylaxis due to multiple medication usage and more pulmonary, vascular, neurologic complications and problems associated with the endotracheal tube during the change of position from lithotomy to prone. During supracostal puncture 
patients with PCNL under regional anaesthesia can follow verbal commands and control respiration for prevention of pulmonary events. The advantages of spinal anaesthesia compared to general anaesthesia were also demonstrated in other procedures such as radical retropubic prostatectomy [12] and unilateral total hip arthroplasty [13].

Recently PCNL under regional spinal anaesthesia was reported to gain benefits because regional spinal anaesthesia achieves better postoperative quality of life due to earlier postoperative recovery but most reports were not part of the controlled study [4,5]. Using SA in PNCL surgery is acceptable and more secure. By faster discharge and reduced recovery time, the patients' quality of life can be improved using SA, which can be a good choice for urologist [14].

Overall, our study demonstrated that SBP, DBP, MAP, and PR in the whole surgery and recovery times did not have any significant difference between 2 groups and that the trend was also somewhat similar in SA and GA, however, patients' hemodynamics were more stable in SA group. Furthermore, bleeding and analgesic demand were significantly higher in GA group. None of the patients needed blood transfusion. These results were similar to other studies demonstrating that SA group had better hemodynamics and lower bleeding during and after the surgery [15-22].

It seems that SA can result in vasodilation and hypotension following sympathetic block. On the other hand, reduced intra-thoracic pressure and epidural vein distension, due to spontaneous ventilation, result in reduced bleeding. Therefore, the results do not seem to be irrational because SA can inhibit stress hormone secretion better than GA.

SA blocks pre-ganglionic sympathetic nerves with many advantages compared to GA, such as redistribution of blood flow to musculoskeletal system, skin, and subcutaneous tissues, as well as reducing SBP, DBP, MAP, and PAP, and better hemostasis. Furthermore, other studies demonstrated better PNCL surgery results, lower blood loss, and lesser side effects (such as nausea, vomiting, and post-op pain) in SA [15, 23].

Among these advantages of SA, decreasing blood loss is a main issue of SA in PCNL surgery. Recent studies investigated the effects of a $200-\mu \mathrm{g}$ of oral clonidine tablet 60 - 90 minutes before anaesthesia, which reduced blood loss significantly in several kinds of surgeries under GA that could be a future choice along with SA in PCNL [24, 25]

Tetzlaff et al. have also shown that in spinal surgeries, SA was a better choice for anaesthesia compared to GA resulting in lower side effects [26].

In another prospective randomized study on PCNL, 52 patients underwent general anaesthesia and 58 patients received spinal anaesthesia. PCNL was performed by standard technique. Intra-operative hypotension, postoperative headache, and low back pain were significantly higher in spinal group but compared to SA, the cost of anaesthetic drugs was more than five times and post-operative analgesic consumption about two times in GA group. Finally authors suggested SA as a safe, effective, and cost-effective method in adult PCNL, the same as our results.

Moreover, in other studies, additional analgesic consumption was reduced in SA group compared to GA group. This may be due to afferent nociceptive block of the spinal cord and faster block of sensory than that of motor nerves $[8,15]$.

In this study, patients with stone in upper pole of kidney, tolerated efficiently, but our sample size was designated for a whole kidney and not solely for upper pole; so because of general concerns about this subtype of kidney stones, future studies are needed with a study population designated for upper pole stones to compare competency and efficacy of SA versus GA.

In view of the results of our study, SA is a faster and safer method of anaesthesia in PNCL surgeries. Using this method can help surgeons to maintain patient in a better hemodynamic and hemostatic state, reduce the GA complications, decrease the need of analgesics, and duration of surgery.

\section{Conclusions}

Percutaneous nephrolithotomy under regional anaesthesia is as effective as PCNL under general anaesthesia. The advantages of regional anaesthesia over general anaesthesia are higher patient satisfaction, less early postoperative pain and less analgesic usage without increasing complications. 
Conflict of Interest- No conflict of interest emerged during the implementation of this work. The paper had not been presented anywhere before.

\section{Funding: Nil, Conflict of interest: None. Permission of IRB: Yes}

\section{References}

1. Stening SG, Bourne S: Supracostal percutaneous nephrolithotomy for upper pole caliceal calculi. J Endourol.1998; 12: 359-62

2. Lojanapiwat B, Prasopsuk S: Upper-pole access for percutaneous nephrolithotomy: comparison of supracostal and infracostal approaches. J Endourol. 2006 ; $20: 491-4$

3. Jun-Ou J, Lojanapiwat B: Supracostal access: does it affect tubeless percutaneous nephrolithotomy efficacy and safety? Int Braz J Urol. 2010; 36: 171-6

4. Kuzgunbay B, Turunc T, Akin S, Ergenoglu P, Aribogan A, Ozkardes H: Percutaneous nephrolithotomy under general versus combined spinalepidural anesthesia. J Endourol. 2009 Nov;23(11):18358. doi: 10.1089/end.2009.0261.

5. Karacalar S, Bilen CY, Sarihasan B, Sarikaya S: Spinal-epidural anesthesia versus general anesthesia in the management of percutaneous nephrolithotripsy. J Endourol. 2009 Oct;23(10):1591-7. doi: 10.1089/end.2009.0224.

6. Singh V, Sinha RJ, Sankhwar SN, Malik A: A prospective randomized study comparing percutaneous nephrolithotomy under combined spinal-epidural anesthesia with percutaneous nephrolithotomy under general anesthesia. Urol Int. 2011;87(3):293-8. doi: 10.1159/000329796. Epub 2011 Sep 14.

7. Urwin SC, Parker MJ, Griffiths R. General versus regional anaesthesia for hip fracture surgery: a metaanalysis of randomized trials. Br J Anaesth. 2000;8 4 (4) : 450-5

8. Indelli PF, Grant SA, Nielsen K, Vail TP. Regional anesthesia in hip surgery. Clin Orthop Relat Res. 2005 : 441: $250-5$

9. Sakura S. [Epidural anesthesia and spinal anesthesia in the elderly]. Masui 2007: 56(2):130-8
10. Ditzler JW, Dumke PR, Harrington JJ, Fox JD. Should spinal anesthesia be used in surgery for herniated intervertebral disk. Anesth Analg. 1959;38(2):118-24

11. Bellman GC, Davidoff R, Candela J, Gerspach J, Kurtz S, Stout L: Tubeless percutaneous renal surgery. J Urol. 1997 May;157(5):1578-82.

12. Salonia A, Suardi N, Crescenti A, Colombo R, Rigatti P, Montorsi F: General versus spinal anesthesia with different forms of sedation in patients undergoing radical retropubic prostatectomy: results of a prospective, randomized study. Int J Urol. 2006 Sep;13(9):1185-90.

13. Maurer SG, Chen AL, Hiebert R, Pereira GC, Di Cesare PE: Comparison of outcomes of using spinal versus general anesthesia in total hip arthroplasty. Am J Orthop (Belle Mead NJ). 2007 Jul;36(7):E101-6.

14. Rozentsveig V, Neulander EZ, Roussabrov E, Schwartz A, Lismer L, Gurevich B, et al. Anesthetic considerations during percutaneous nephrolithotomy. J Clin Anesth. 2007;19(5):351-5

15. Covino BG. Rationale for spinal anesthesia. Int Anesthesiol Clin. 1989; 27(1) :8-12

16.Davis S, Erskine R, James MF. A comparison of spinal and epidural anaesthesia for hip arthroplasty. Can J Anaesth. 1992;39(6):551-4

17. Guedj P, Eldor J, Gozal Y. [Comparative study of conventional spinal anesthesia and combined spinalepidural anesthesia in gynecological surgery]. Ann Fr Anesth Reanim. 1992;11(4):399-404

18. Holmstrom B, Laugaland K, Rawal N, Hallberg S. Combined spinal epidural block versus spinal and epidural block for orthopaedic surgery. Can J Anaesth. 1993;40(7):601-6

19. Niemi TT, Pitkanen M, Syrjala M, Rosenberg PH. Comparison of hypotensive epidural anaesthesia and spinal anaesthesia on blood loss and coagulation during and after total hip arthroplasty. Acta Anaesthesiol Scand. 2000;44(4):457-64

20. Seeberger MD, Lang ML, Drewe J, Schneider M, Hauser E, Hruby J. Comparison of spinal and epidural 
anesthesia for patients younger than 50 years of age. Anesth Analg.1994;78(4):667-73

21. Stober HD, Mencke T. [General anesthesia or spinal anesthesia for hip prosthesis replacement? Studies of acceptance of both procedures by patients]. Anaesthesiol Reanim 1999;24(6):151-6

22. Sutter PA, Gamulin Z, Forster A. Comparison of continuous spinal and continuous epidural anaesthesia for lower limb surgery in elderly patients. A retrospective study. Anaesthesia.1989;44(1):47-50

23. Kehlet $H$. The stress response to surgery: release mechanisms and the modifying effect of pain relief. Acta Chir Scand Suppl. 1989;550:22-8
24. McLain RF, Kalfas I, Bell GR, Tetzlaff JE, Yoon HJ, Rana M. Comparison of spinal and general anesthesia in lumbar laminectomy surgery: a casecontrolled analysis of 400 patients. J Neurosurg Spine. 2005;2(1):17-22

25. Ebneshahidi A, Mohseni M. Premedication with oral clonidine decreases intraoperative bleeding and provides hemodynamic stability in cesarean section. Anesth Pain. 2011:1(1):30-3

26. Tetzlaff JE, Dilger JA, Kodsy M, al-Bataineh J, Yoon HJ, Bell GR. Spinal anesthesia for elective lumbar spine surgery. J Clin Anesth. 1998;10(8):666-9

\section{How to cite this article?}

Wani Z, Harkawat D.K., Sharma M. Spinal versus general anaesthesia in percutaneous nephrolithotomy. Int J Med Res Rev 2017;5(01):26-31. doi:10.17511/ijmrr. 2017.i01.04 